\title{
Pointing effects and their consequences for Spitzer IRAC exoplanet observations
}

\author{
Carl J. Grillmair ${ }^{\mathrm{a}}$, Sean J. Carey ${ }^{\mathrm{a}}$, John R. Stauffer ${ }^{\mathrm{a}}$, Mark E. Fisher ${ }^{\mathrm{b}}$, Ryan Olds ${ }^{\mathrm{b}}$, James G. Ingalls ${ }^{\mathrm{a}}$, \\ Jessica E. Krick ${ }^{\mathrm{a}}$, William J. Glaccum ${ }^{\mathrm{a}}$, Seppo Laine ${ }^{\mathrm{a}}$, Patrick J. Lowrance, Jason A. Surace ${ }^{\mathrm{a}}$ \\ ${ }^{a}$ Spitzer Science Center, California Institute of Technology, Mail Stop 220-6, 1200 E. California \\ Blvd., Pasadena, CA, USA 91125, 'ockheed Martin Space Systems, 12257 S. Wadsworth Blvd., \\ Littleton, CO, USA, 80127
}

\begin{abstract}
Spitzer observations of exoplanets routinely yield accuracies of better than one part in 10,000. However, there remain a number of issues that limit the attainable precision, particularly for long duration observations. These include initial pointing inaccuracies, pointing wobble, initial target drift, long-term pointing drifts, and low and high frequency jitter. Coupled with small scale, intrapixel sensitivity variations, all of these pointing issues have the potential to produce significant, correlated photometric noise. We examine each of these issues in turn, discussing their suspected causes and consequences, and describing possible and planned mitigation techniques.
\end{abstract}

Keywords: Spitzer Space Telescope, Exoplanets

\section{INTRODUCTION}

While the Spitzer Space Telescope offers astronomers enormous gains in sensitivity over all previous infrared telescopes, it was not designed with high precision observations of exoplanets in mind. Nonetheless, the spacecraft has significantly exceeded its design requirements, and Spitzer observations with the Infrared Array Camera (IRAC) typically reach to within $<50 \%$ of the photon-noise limit. Precisions of $100 \mathrm{ppm}$ are routinely reached, and 30-60 ppm have recently been achieved ${ }^{1,2}$. This level of photometric precision enables a wide range of exoplanet validation and characterization, from measuring planetary radii to characterizing atmospheric compositions and measuring wind and thermal profiles. Over $25 \%$ of the allocated observing time in cycles 7 and 8 of Spitzer's post-cryogenic, "warm" mission have been devoted to science requiring very high precision relative photometry (either exoplanet validation and characterization or investigations of cloud cover and weather for brown dwarfs).

Extracting high-precision photometry from IRAC data is complicated by sources of correlated noise that must be trended from the data. The most significant source of correlated noise is variations in the gain across individual detector pixels coupled to small telescope motions during the long staring observations used for exoplanet science. Figure 1 shows an intrapixel sensitivity variation or "pixel phase" calibration map (p-map) generated using many thousands of individual images, mapping relative sensitivities on a sub-pixel scale ${ }^{3}$. These gain variations are not resolved measurements of the change in gain across a single individual pixel, but rather are the net effect of moving a somewhat undersampled optical point response function with a full-width-at-half-maximum of $\sim 1.4$ " over $\sim 30$ pixels within a 3 pixel radius aperture used to estimate the total source brightness.

Any motion of a stellar image on the scale of Figure 1 will induce commensurate fluctuations (at the level of fractions of a percent at sub-pixel scales and up to $2 \%$ across a pixel) in the measured flux. While it is both possible and effective to correct for these fluctuations using a $\mathrm{p}$ - $\mathrm{map}^{4}$, the p-map itself is subject to unavoidable measurement errors. Moreover, observations of a variety of sources show that there are significant non-linearities at play: e.g., the relative sensitivities depend not only on the sub-pixel position of the source, but also on the number of photons collected. Linearity corrections are currently in development, but given the finite amount of calibration time available, they will necessarily also be subject to uncertainties. Achieving the best possible photometric precision therefore requires that target images be placed accurately on the detector, and that subsequent movement of the image be minimized. There are several known issues with spacecraft pointing that complicate this ideal, including target placement accuracy, pointing wobble, initial pointing drifts, long-term drifts, and pointing jitter. We discuss each of these effects in turn below. 

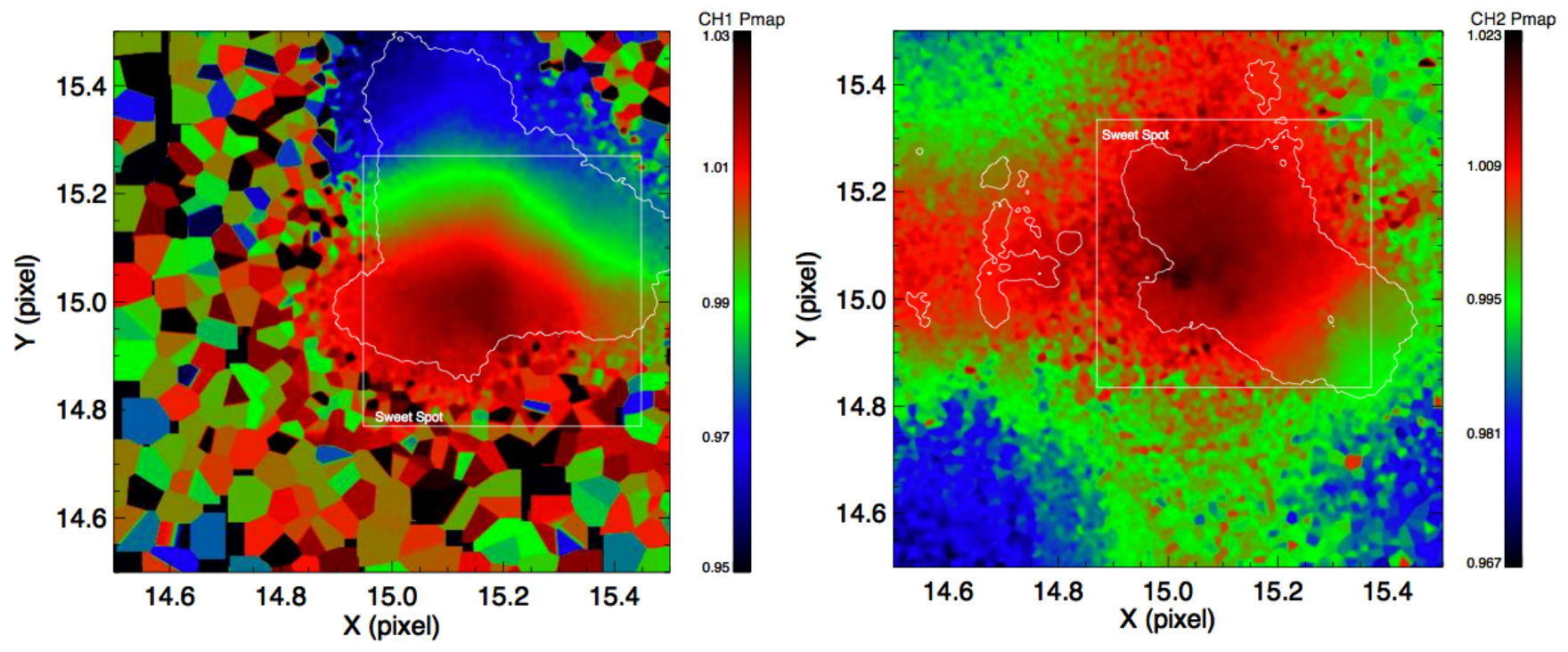

Figure 1. Phase maps for the central pixels of the subarrays for the 3.6 micron and 4.5 micron detectors. Coordinates are such that 15.0,15.0 denotes the center of the pixel, while 15.5, 15.5 denotes the upper right hand corner.

\section{POINTING ACCURACY}

The development of pixel phase maps, characterizing the sensitivity of selected pixels to a resolution of roughly 0.02 pixels, has demonstrated the need for accurate target placement on the detectors. The p-maps show structure on a scale of 0.05 pixels, and there are regions that are significantly more uniform in terms of sensitivity than others. Even if pointing variations could be reduced to the limiting values induced by jitter (see below), the p-map in Figure 1 shows that it would be much better to be near the centers of the pixels than near the edges. Spitzer's blind pointing accuracy of $0.3 \operatorname{arcsec}(1 \sigma$ radial) is insufficient to ensure that the target routinely lands within the $0.5 \times 0.5 \operatorname{arcsec}$ pixel sweet spot near the center of the pixel, and not on the steeper gradients towards the edges. Another consideration is that p-maps in Figure 1 required $\sim 90$ hours of telescope time to develop. Generating much larger p-maps that could accommodate blind pointing would require a prohibitive investment of observing time.

To ensure that the target lands on the preferred portion of a pixel, observations requiring high precision photometry should make use of Pointing and Calibration Reference Center (PCRS) peak-ups whenever possible ${ }^{5}$. The PCRS can measure pointing offsets to an accuracy of better than 0.1 arcsec for targets or offset reference stars with visual magnitudes from 7 to 12.5 . Tests with PCRS peak-ups have shown pointing repeatability of $0.07 \operatorname{arcsec}(1 \sigma$ radial), with better than $94 \%$ of all centroids falling within the $0.5 \times 0.5$ arcsec p-map sweet spot. The additional pointing accuracy afforded by PCRS peak-ups ensures that observations at multiple epochs will have similar systematics. This will make it easier to identify real astrophysical phenomena, and to coadd multiple epochs to improve signal-to-noise ratio.

\section{POINTING WOBBLE}

Figure 2 shows the measured $\mathrm{x}$ and y positions of HD 290458 over the course of nearly a day of continuous observations. Apparent are pronounced oscillations in the centroid position with an amplitude of 0.15 arcsec and a period of about an hour. This "sawtooth" has long been recognized ${ }^{6}$ and, by virtue of having a period similar to the transit durations of many exoplanets, has complicated both photometric and spectral observations of such systems. The source of this sawtooth has been traced to the periodic cycling of a survival heater for a battery within the spacecraft. The suspected physical cause of the pointing wobble is a thermo-mechanical expansion of the spacecraft assembly correlated with the heater cycling. 


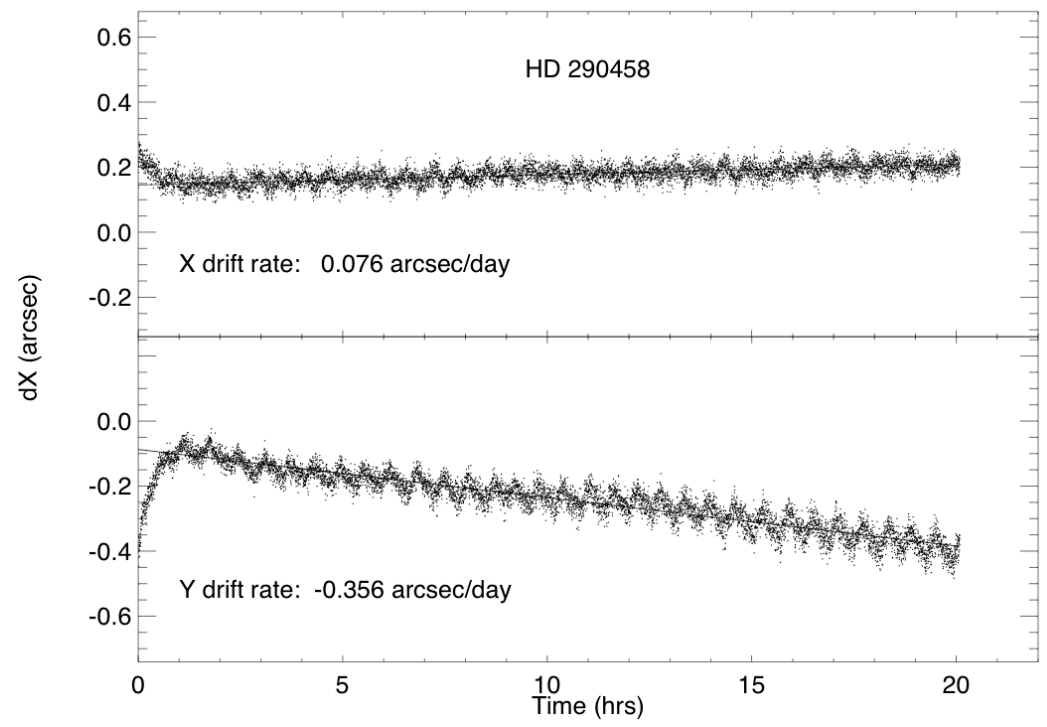

Figure 2. X- and y-axis centroid offsets for a 20-hour-long, phase-curve observation of HD 209458 in channel 1 (3.6 $\mu \mathrm{m})$.

The excess heat causes thermal bending of spacecraft structures and very small changes in alignment between the star trackers and the telescope bore sight. Combining this pointing wobble with measured intra-pixel sensitivity variations can, without correction, yield peak-to-peak flux fluctuations of up to $\sim 5 \%$.

Recognizing the significance of this pointing wobble for high-precision photometry, a mitigation strategy was developed to reduce the cycling time of the survival heater. By reducing the cycling time, the period of the oscillation would be moved further away from the characteristic transit durations for exoplanets, and the amplitude of the signal would presumably be reduced as well. The heater cycling was initially set to $2 \mathrm{~K}$ (range of 4 data numbers) as was common in heritage systems. A thermal analysis revealed no issues with reducing the cycling range to $2 \mathrm{DNs}$ or $1 \mathrm{~K}$. As of 17 Oct 2010 , the heater cycling was set to the $1 \mathrm{~K}$ range. Figures 3 and 4 show the results of this strategy. The mean period of the wobble has been reduced to 39 minutes, and the mean amplitude of the oscillation has been reduced by a factor of 2 .

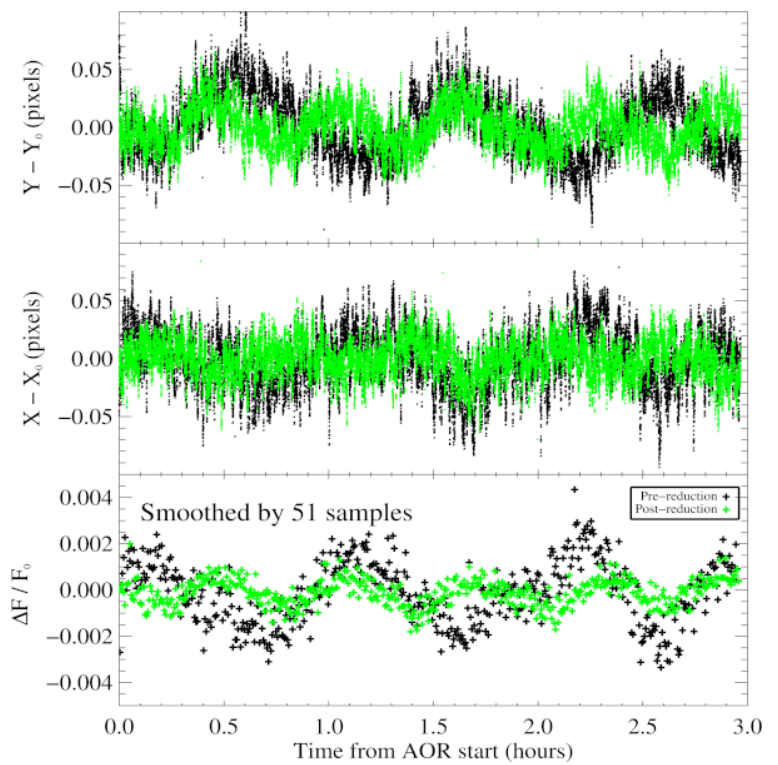

Figure 3. The pointing wobble or "sawtooth", before (black) and after (green) increasing the battery heater cycling frequency. Top: Y position vs. time, middle: X position vs. time, bottom: identically binned flux measurements, showing the reduction in the amplitude of flux variations attributed to the pointing wobble. 
While the pointing wobble has been substantially reduced, it remains a significant contributor to the error budget. The sawtooth form of the oscillation has variously been modeled as sinusoidal, or with increasing and decreasing linear trends of fixed length, with mean slopes and turn-over phases measured for each observing request. However, even a cursory examination of Figures 2 and 3 shows that the amplitude, period, and form of the sawtooth are not precisely constant from one heating cycle to the next. Time averaged fitting residuals are typically on the order of $0.05 \%$ from cycle to cycle. While this is less important than the photon noise per observation, it means that pointing wobble could remain a somewhat problematic systematic on short timescales (e.g., transits).

Figure 4 shows power spectra for $\mathrm{x}$ and $\mathrm{y}$ centroid motions for 34 long-duration (10-22 hours), phase curve observations of exoplanet systems taken after October 2010. Periodograms were generated using the Lomb-Scargle algorithm for each individual sequence of observations, resampled to a common frequency scale, and then averaged per frequency. The relatively sharp peak at $\sim 39$ minutes corresponds to the current, higher frequency pointing wobble, while the smaller peak at 18 minutes is its first harmonic. These peaks are not perfectly sharp, due both to the non-sinusoidal shape of the pointing wobble, and to a 2-3 minute dispersion in the measured wobble periods. It remains unclear what is causing this dispersion in the period. While it seems plausible that solar insolation, varying as a function of telescope pitch angle, may trip the survival heater more or less frequently, we have found instances where the period, shape, and amplitude of the wobble change noticeably between successive, identical, back-to-back visits to the same target, or even within a single long observing request.

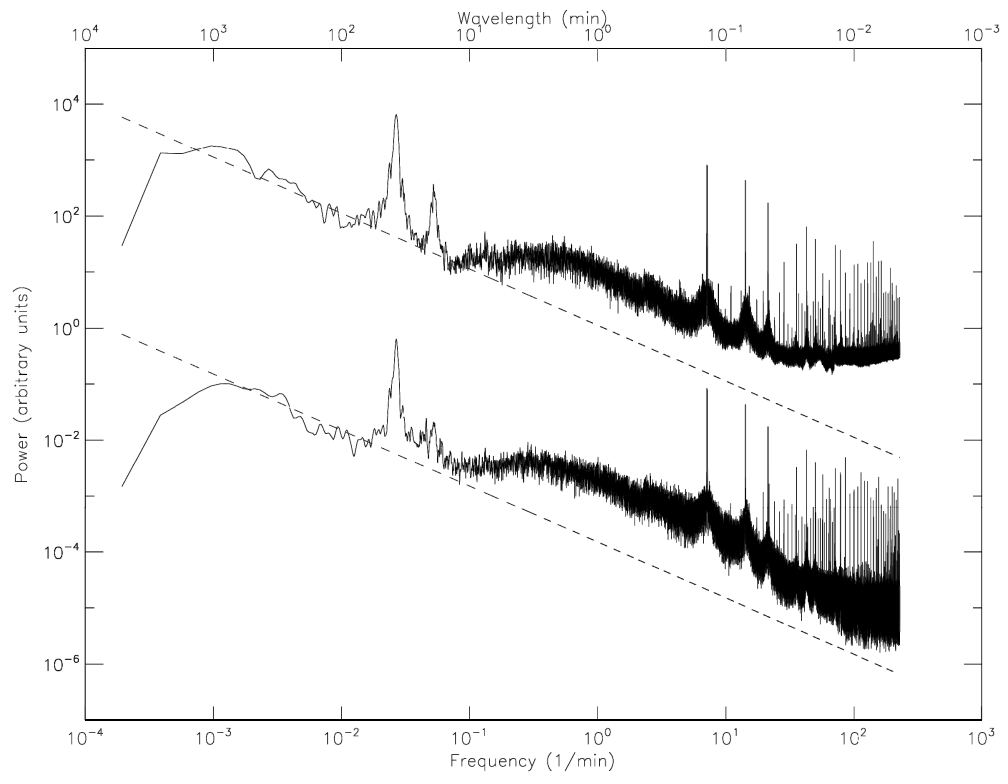

Figure 4. Power spectra of $\mathrm{x}$ (lower) and y (upper) centroid motions, averaged over 34 long (8-22 hour) observing sequences. The peaks at 39 and 18 minutes correspond to the current battery heater cycle time and corresponding pointing wobble. The dashed lines show the run of $1 / \mathrm{f}$ noise for comparison. 
Additional peaks, some of which appear to be harmonics, are apparent on timescales of 8 seconds or less. These peaks are not fully understood at present and are being investigated. The power spectra as a whole show the characteristics of 1/f noise, though there appears to be comparatively more power than expected in a broad bump centered near 2.5 minutes (see below). The power spectrum appears to flatten to towards white noise beyond $\sim 50$ cycles per minute $(\sim 1$ sec).

\section{POINTING DRIFTS}

\subsection{Initial pointing drifts}

Observations sometimes show an initial drift in the telescope pointing, irrespective of whether PCRS peak-ups are carried out prior to initiating the sequence. This approximately linear drift varies from 0 to $0.5 \operatorname{arcsec}$ over periods ranging from 30 to 60 minutes, with drifts along the y-axis generally being larger and of the opposite sign to those along the $\mathrm{x}$-axis (see Figure 2 for an example). Where observations of the same target were carried out in succession, the subsequent observations did not show this initial drift, indicating that this is not a random occurrence. While observers often simply discard the obviously affected data, an important secondary consequence of the initial drift is that the target can drift away from the well-characterized, most uniform region of the pixel where a PCRS peak-up would initially place it.

Preliminary investigation suggests that the initial drift occurs when an observation was preceded by a relatively large slew across the sky, indicating that drift may be due to significant changes in the pitch angle of the telescope. Pitch angle is defined as P.A. $=\Theta-90$, and $\Theta$ is the angle between the telescope bore sight and the sun. While the solar panels completely shadow the telescope for P.A. $\approx 0^{\circ}$, sunlight impinges on the spacecraft's high gain antenna assembly for P.A. $>0^{\circ}$. We are currently examining the hypothesis that this additional heat load could produce thermal stresses and flexure in the telescope structure that would slightly alter the alignment between the star trackers and the telescope bore sight (in a manner similar to that for the battery heater above).

Figure 5 shows the magnitude of the initial drift as a function of the change in P.A. for a sample of 118 observations of exoplanets taken over the course of the warm mission. The data show a clear correlation, with the effect being most pronounced in the Y direction. Pending further onboard testing, we conclude that initial pointing drift is primarily due to large changes in pitch angle. Knowing this, one could, in principal, schedule Spitzer observations in such a way that high photometric precision observations are always preceded by observations of targets with very nearly the same pitch angle. However, with a limited number of available targets and the many other constraints and sometimes conflicting scheduling requirements, it was ultimately deemed more expedient to simply point the telescope at the exoplanet in question for 30 minutes prior to beginning the observation. This additional settling time will not be deducted from the time allocated to observers in Cycle 7 or 8 . However, observers in future cycles will need to include this additional time in their proposal requests. With this procedure in place, we expect the initial drift problem will be inconsequential for high-precision photometric time series. 


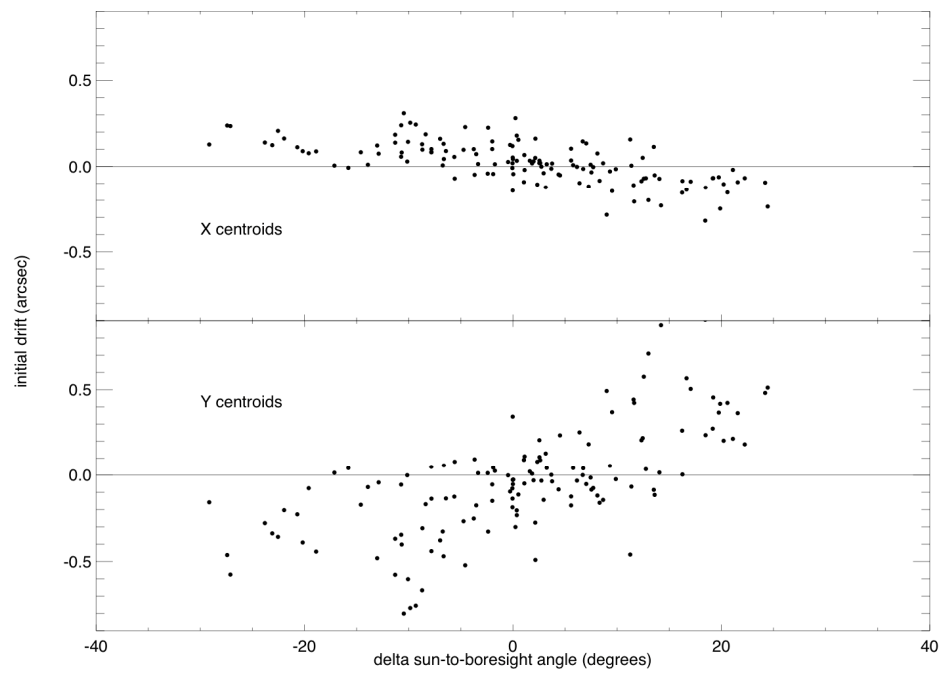

Figure 5. Initial drifts of $\mathrm{x}$ - and $\mathrm{y}$-centroids in 118 exoplanet observing sequences versus the mean change in pitch angle of the telescope from targets observed during the preceding half hour. There are clear correlations in both axes, with the effect being stronger and of opposite sign in the y-axis.

\subsection{Long-term pointing drifts}

Visible in Figure 2 is a long-term change, extending from 1 to 20 hours, in the average y-centroid position of a star on the IRAC detector. This effect is seen in about $80 \%$ of photometric sequences over 10 hours in length. The effect has two potentially significant consequences: i) the drift can cause the star+planet to move away from the preferred, wellcharacterized, and uniform-sensitivity portion of a pixel, and ii) the observed photometric signal may mask real changes in the light output of star+planet, or produce variations which are purely spurious. If the goal of an observation is to measure the phase curve of a tidally-locked planet - that is, the long-term variation in the light output of the planet as it presents different longitudes at different illumination angles - then imperfect p-map corrections may either overwhelm subtle but real variations in the source brightness, or generate modulations that could be misinterpreted as being due to temperature variations in the planetary atmosphere.

One suspected cause of the long-term pointing drift is a difference in the way that differential velocity aberration corrections are handled by the spacecraft's Command and Data Handling computer (C\&DH) and by the star trackers. Velocity aberration causes targets to appear up to 20 arcsec away from their true positions on the sky, depending on the angle between the sight line to the star and Spitzer's velocity vector. As the telescope moves along its orbit, its velocity vector is changing at a rate of roughly $1^{\circ}$ per day. The use of a fixed velocity aberration correction per observation request by the $\mathrm{C} \& \mathrm{DH}$ and a frequently updated correction by the star trackers leads to a constant correction signal in the pointing control system. This correction signal generates a linear drift rate in the negative $\mathrm{Y}$ direction on the IRAC detectors that ranges from -0.31 arcsec/day near the outer limit of Spitzer's operational pointing zone (OPZ), to -0.36 arcsec/day near the inner, sunward limit of the OPZ.

Experiments using Spitzer's ability to track moving objects were conducted to determine whether the long term drift could be corrected by commanding a tracking rate of equal magnitude but opposite sign. The experiment proved unsuccessful, primarily because the spacecraft must then rely almost entirely on its onboard gyros, which themselves are subject to drift on relatively short timescales. An alternative mitigation strategy currently under study would effectively prevent differential velocity aberration corrections from being applied over the course of long photometric time series. 
Closer examination of Figure 2 shows that y drift, while agreeing well with the -0.35 arcsec/day value expected, is not perfectly linear, showing higher-order undulations on the scale of hours. Moreover, the measured x-drift is not zero, as one would expect if the differential velocity aberration correction were the only long-term drift issue. Indeed, Figure 6 shows an unexpected but significant correlation between the magnitude of the drift in the $\mathrm{X}$ direction and the pitch angle of the telescope. Since the pitch angle alone determines how much sunlight falls on the high gain antenna assembly, we suspect once again that thermal stresses and flexure may be at work. For a sample of 137 staring observations more than 12 hours in length, the long-term drifts show dispersions of 0.10 and 0.18 arcsec/day in $\mathrm{x}$ and $\mathrm{y}$, respectively, with $4 \%$ of the sample showing positive y drifts. While the mean y drift $(-0.31 \mathrm{arcsec} /$ day $)$ is in accord with expectation, there is clearly more work to be done to fully understand these long-term drifts.
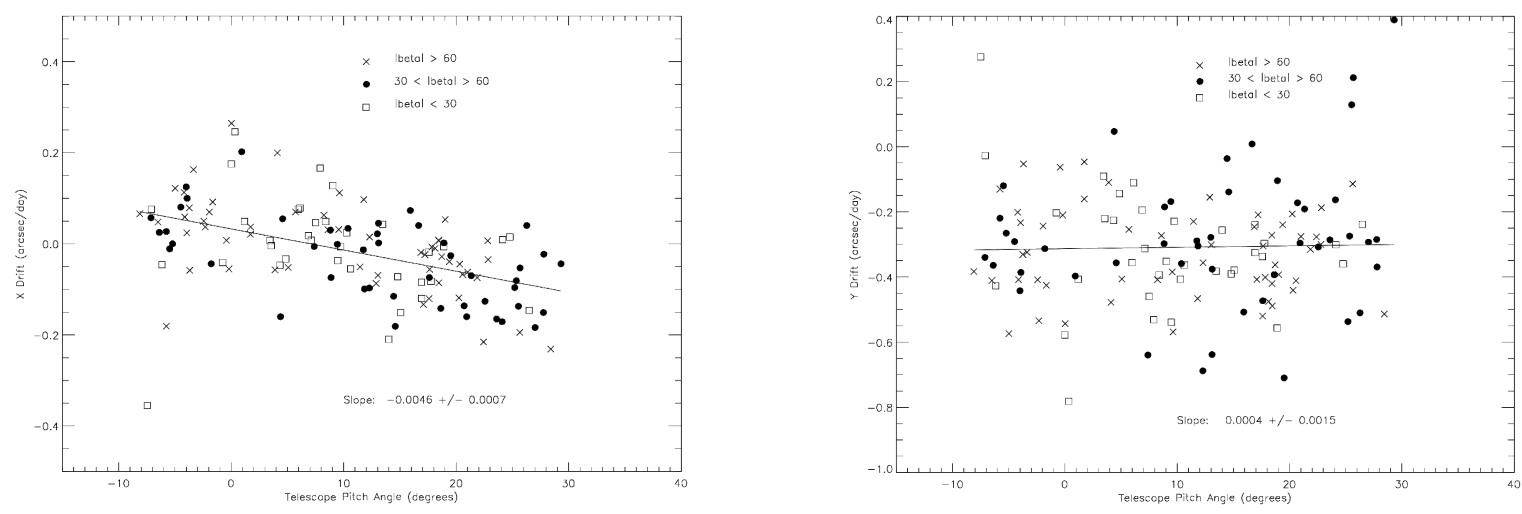

Figure 6. Long term drift in the $\mathrm{x}$ and $\mathrm{y}$ centroids for 137 staring observations vs. telescope pitch angle. A pitch angle of $0^{\circ}$ indicates an angle between the sun and the telescope bore sight of $90^{\circ}$. Spitzer's operational pointing zone lies between pitch angles of roughly $-10^{\circ}$ and $+30^{\circ}$. Different symbols indicate targets having different ranges of ecliptic latitude.

While the long-term drift is generally fairly uniform in rate and direction once established, the consequences for timeseries photometry may not always be so, depending on the regions of a pixel over which the target image passes. Very long sequences (e.g,. $>12$ hours) are therefore inadvisable for observations requiring the highest possible photometric accuracy. We recommend breaking long observing requests into sequences of no longer than 12 hours, and using PCRS peak-ups to return as nearly as possible to the preferred pixel location. Preliminary testing shows that this procedure is working well to keep the target in the sweet spot. This procedure necessarily creates discontinuities in orbit-spanning, phase curve sequences, but keeps the target always within the well-characterized and most uniform portion of the pixel. P-map corrections will then enable successive sequences to be brought back to a common system.

\section{JITTER}

Figure 7 shows $\mathrm{x}$ and $\mathrm{y}$ centroid positions measured at 0.02 second intervals for several long staring sequences. The centroids show correlated undulations with peak-to-peak amplitudes ranging from 0.03 to 0.1 arcseconds, and periods ranging from 1 to 3 minutes. This range and spread of periods appears to be consistent with the broad bump in the power spectrum of Figure 4, though these undulations remain under investigation and are not yet fully understood.

Previous analyses have shown evidence of $\sim 0.03$ arcsec motions at frequencies of $>50 \mathrm{~Hz}$. We broadly categorize both these motions and the undulations in Figure 7 as telescope "jitter", with both low and high frequency components. Possible causes are many, and may include noise in the star tracker solutions, momentum variations in the reaction wheel assembly harmonically coupled to the spacecraft structure, and even micrometeoroid impacts. We suspect that there is little prospect of being able to reduce or eliminate these motions during the remainder of the Spitzer mission, 
though our efforts to understand them continue. We are reasonably confidant that at least the lower frequency, higher amplitude motions will be largely mitigated by application of the P-maps. The highest frequency $(>50 \mathrm{~Hz})$ motions will continue to set a noise floor for bright targets.
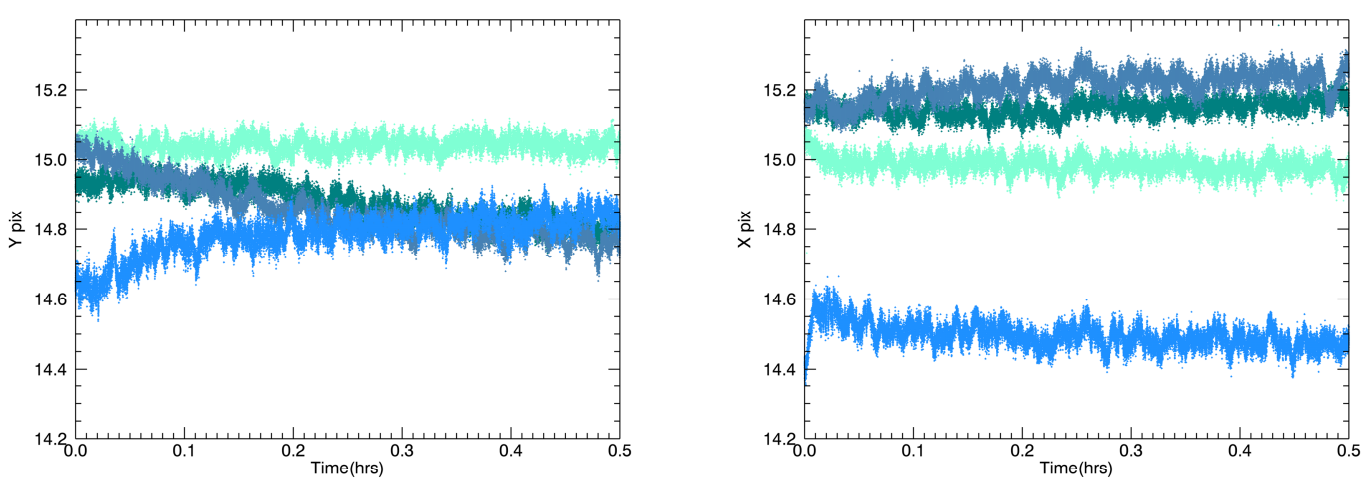

Figure 7. $\mathrm{X}$ and $\mathrm{y}$ centroids as a function of time for four exoplanet observations, taken at $0.02 \mathrm{sec}$ intervals, showing the effects of low frequency telescope jitter.

\section{SUMMARY}

While performing beyond its design requirements, the Spitzer Space Telescope is subject to a number of pointing issues that may limit the photometric precision available for the most demanding, high-accuracy observations of transiting exoplanets. Table 1 summarizes these effects, along with their currently characteristic amplitudes and timescales. Some of these effects can or have been significantly reduced in their severity through flight software modifications (wobble), scheduling (initial drift), and recommended observing practices (pointing accuracy, long-term drift). Telescope jitter will continue to set a limit on the attainable precision for bright stars. While we expect that application of the latest pixel phase maps will significantly reduce the photometric consequences of such residual motions, our efforts to identify, understand, and perhaps mitigate these motions will continue.

Table 1. Current amplitudes and timescales for identified Spitzer pointing effects.

$\begin{array}{lll}\text { Pointing effect } & \text { Characteristic amplitude } & \text { Characteristic timescale } \\ \text { Accuracy } & 0.07 \operatorname{arcsec} & \text { N/A } \\ \text { Wobble } & 0.07 \operatorname{arcsec} & \sim 40 \text { minutes } \\ \text { Initial drift } & 0.5 \operatorname{arcsec} & \sim 30 \text { minutes } \\ \text { Long-term drift } & 0.3 \operatorname{arcsec} & \sim 1 \text { day } \\ \text { Low frequency jitter } & 0.07 \operatorname{arcsec} & \sim 2 \text { minutes } \\ \text { High frequency jitter } & 0.03 \operatorname{arcsec} & <0.02 \text { seconds }\end{array}$




\section{REFERENCES}

[1] Demory et al., "Detection of a transit of the super-Earth 55 Cancri e with warm Spitzer", Astronomy \& Astrophysics, 533, 114-120 (2011).

[2] Todorov et al., "Warm Spitzer Observations of Three Hot Exoplanets: XO-4b, HAT-P-6b, and HAT-P-8b", The Astrophysical Journal, 746, 111-123 (2012).

[3] Ingalls, J. G., Krick, J. E., Carey, S. J., Laine, S., Surace, J. A., Glaccum, W. J., Grillmair, C. J., and Lowrance, P. J., "Intra-pixel gain variations and high-precision photometry with the infrared array camera (IRAC)", Proc. SPIE 8442-68 (2012).

[4] Ballard, S., Charbonneau, D., Deming, D., Knutson, H. A., Christiansen, J. L., Holman, M. J., Fabrycky, D., Seager, S., A'Hearn, M. F., "A Search for a Sub-Earth-Sized Companion to GJ 436 and a Novel Method to Calibrate Warm Spitzer IRAC Observations", Publications of the Astronomical Society of the Pacific, 122, 1341-1352 (2010).

[5] Krick, J., Ingalls, J., Carey, S., and von Braun, K. "A New Spitzer IRAC Technique to Characterize Exoplanet Atmospheres", American Astronomical Society, Meeting \#220, \#505.01 (2012).

[6] Morales-Calderon, M. et al., "A Sensitive Search for Variability in Late L Dwarfs: The Quest for Weather", The Astrophysical Journal, 653, 1454-1463 (2006) 